\title{
Área foliar de mudas de urucum (Bixa orellana L.) estimada por diferentes métodos: uma análise comparativa
}

\author{
Leaf area of seedlings of urucum (Bixa orellana L.) estimated by different \\ methods: a comparative analysis
}

\author{
Fernanda da Silva Pinheiro ${ }^{\mathrm{I}}$, Gustavo Bastos Lyra ${ }^{\mathrm{II}}$, \\ Marcel Carvalho Abreu ${ }^{\text {III }}$, José Carlos Arthur Junioriv, \\ Leonardo Duarte Batista da Silva ${ }^{\mathrm{v}}$, Guilherme Bastos Lyra ${ }^{\mathrm{VI}}$, \\ Ednaldo Oliveira dos Santos ${ }^{\mathrm{VII}}$
}

\section{Resumo}

A determinação da área foliar (AF) das culturas é um parâmetro indicativo do crescimento e da produtividade, sendo a busca por métodos precisos e exatos para a sua estimativa de extrema importância. O urucum (Bixa orellana L.) é uma espécie arbórea nativa da Floresta Amazônica e da Mata Atlântica, cuja importância econômica está relacionada ao corante natural extraído de suas sementes. Assim, o presente estudo objetivou comparar diferentes métodos para determinação da AF em mudas de urucum. Para isso, o método planimétrico foi considerado padrão e, com base nele, testou-se a equivalência em relação aos métodos das dimensões foliares - comprimento $(C)$ e largura $(\mathrm{L})$ - e ao scanner. No método das dimensões foliares o fator de forma (f) de 0,64 foi ajustado previamente para o urucum por meio da análise de regressão entre $\mathrm{AF}$ e o produto $\mathrm{C}$ x L. No método do scanner, utilizou-se o aplicativo ImageJ para determinar a AF. A regressão linear sem intercepto entre AF do método padrão e AF estimada por cada método alternativo foi utilizada para verificar a concordância dos resultados. Os métodos das dimensões foliares e do scanner foram equivalentes ao método padrão ( $5 \%$ de probabilidade de erro) e equivalentes entre si ( $1 \%$ de probabilidade de erro). Assim, verificou-se que esses métodos podem ser empregados com segurança na estimativa da área foliar do urucum. Contudo, o método das dimensões apresenta vantagens práticas em relação aos outros por ser não destrutivo.

Palavras-chave: Método não destrutivo; Método direto; Modelagem da lâmina foliar; Análise de regressão

\footnotetext{
Engenheira Florestal, Pesquisadora Autônoma, Av. Dr. Arruda Negreiros, 1445, Nova América, CEP 26023-210, Nova Iguaçu (RJ), Brasil. nandaah_ pinheirooh@hotmail.com (ORCID: 0000-0002-1183-9421)

II Meteorologista, Dr., Professor do Departamento de Ciências Ambientais, Instituto de Florestas, Universidade Federal Rural do Rio de Janeiro, Rod. BR 465, Km 07, CEP 23890-000, Seropédica (RJ), Brasil. gblyra@gmail.com (ORCID: 0000-0002-9882-7000)

III Engenheiro Florestal, Dr., Professor do Departamento de Ciências Ambientais, Instituto de Florestas, Universidade Federal Rural do Rio de Janeiro, Rod. BR 465, Km 07, CEP 23890-000, Seropédica (RJ), Brasil. marcelc.abreu@gmail.com (ORCID: 0000-0002-6457-421X)

IV Engenheiro Florestal, Dr., Professor do Departamento de Silvicultura, Instituto de Florestas, Universidade Federal Rural do Rio de Janeiro, Rod. BR 465, Km 07, CEP 23890-000, Seropédica (RJ), Brasil. josecarlosarthurjunior@gmail.com (ORCID: 0000-0002-4161-8822)

Engenheiro Agrícola, Dr., Professor do Departamento de Engenharia, Instituto de Tecnologia, Universidade Federal Rural do Rio de Janeiro, Rod. BR 465, Km 07, CEP 23890-000, Seropédica (RJ), Brasil. monitoreambiental@gmail.com (ORCID: 0000-0001-9082-7965)

vi Engenheiro Agrônomo, Dr., Professor do Centro de Ciências Agrárias, Universidade Federal de Alagoas, BR-104, Km 85, s/n, CEP 57100-000, Rio Largo (AL), Brasil. gbastoslyra@gmail.com (ORCID: 0000-0002-6186-2048)

VII Meteorologista, Dr., Professor do Departamento de Ciências Ambientais, Instituto de Florestas, Universidade Federal Rural do Rio de Janeiro, Rod. BR 465, Km 07, CEP 23890-000, Seropédica (RJ), Brasil. edmeteoro@ hotmail.com (ORCID: 0000-0001-6217-205X)
} 


\begin{abstract}
Leaf area (LA) determination of the crops is a parameter indicative of growth and yield, and precise and accurate methods for its estimation are very important. Urucum (Bixa orellana L.) is an arboreal species, native to the Amazon Rainforest and Atlantic Forest, with economic importance due to its natural dye seed extracts. Thus, the aim of this paper is to compare different methods for LA determination in Urucum seedlings. To do so, the planimetric method was considered the standard method and its equivalence to the method based in leaf size parameters - length (L) and width (W) - and the scanner method were tested. In the method based in leaf size parameters, the $\mathrm{f}$ of 0.64 was previously adjusted for the annatto by regression analysis between LA and the $\mathrm{C} x \mathrm{~L}$ product. In the scanner method, the ImageJ app was used to determine the LA. Linear regression without intercept between LA through the standard method and LA estimated through each alternative method was used to check the conformity between the results. Leaf size and scanner methods were equivalent to the standard method (5\% error probability) and equivalent to each other ( $1 \%$ error probability). Thus, these methods can be reliably used for estimating the Urucum leaf area. However, the dimension method has practical advantages compared to the others as it is a nondestructive method.
\end{abstract}

Keywords: Non-destructive method; Direct method; Leaf blade modeling; Regression analysis

\title{
Introdução
}

O urucum (Bixa orellana L.) é uma espécie nativa da Floresta Amazônica e da Mata Atlântica (ARAUJO NETO et al., 2018) conhecida pelo corante natural extraído de suas sementes. O corante do urucum varia entre as cores amarela, marrom e vermelha (FARIA et al., 2019) e tem grande valor econômico e industrial (FONTANA et al., 2016). Mundialmente, o urucum se destaca por ser a principal fonte de corante natural (KISSMANN; SCALON; TEODOSIO, 2013). A posição de destaque se consolidou especialmente após a restrição imposta pelos países europeus e EUA aos corantes artificiais utilizados nas indústrias de alimentos e de cosméticos.

O urucuzeiro pode ainda ser usado como planta ornamental e para a recuperação de áreas degradadas, devido ao rápido crescimento da espécie (KISSMANN; SCALON; TEODOSIO, 2013) e à baixa exigência em relação a tratos culturais. Contudo, procedimentos para a produção de mudas de urucum são escassos (ARAUJO NETO et al., 2018).

A produção de mudas de qualidade está diretamente relacionada ao sucesso do plantio e ao assentamento das plantas no campo. Portanto, o padrão de crescimento das mudas no viveiro, por meio da observação de seus parâmetros fisiológicos e morfológicos, é de extrema importância. A área foliar (AF) é um parâmetro de destaque como indicativo do crescimento (FASCELLA; DARWICH; ROUPHAEL, 2013; ABREU et al., 2015) e da produtividade (KUMAR et al., 2017; LIU et al., 2017) de culturas. Além disso, a AF tem uma relação direta com a quantidade de energia luminosa interceptada e a conversão desta em energia química na fotossíntese (FAVARIN et al., 2002; SALA et al., 2015). Dessa forma, informações sobre a AF podem ser associadas com o crescimento e o desenvolvimento vegetal, com a eficiência fotossintética e com a transpiração vegetal (MARTINS et al., 2008; ABREU et al., 2015).

A AF é influenciada diretamente pela fertilização, irrigação (KANDIANNAN et al., 2009) e pelo ataque de doenças e/ou pragas (MORGADO et al., 2013; OLIVEIRA et al., 2017). Por meio da AF é possível determinar diversos índices, por exemplo, o índice de área foliar (IAF), a razão área foliar/produção, a relação entre a área foliar e a superfície foliar exposta e a área foliar específica (SALA et al., 2015). Esses índices são usados em análise de crescimento, em modelagem e auxiliam no projeto e no manejo das práticas relacionadas às atividades supracitadas.

Existem diversos métodos para a determinação da AF, que podem ser classificados como diretos ou indiretos e, ainda, métodos destrutivos ou não destrutivos (KERAMATLOU et al., 2015). Os métodos diretos, apesar de serem mais exatos, exigem intervenções realizadas diretamente na folha, sendo de difícil utilização devido à demanda de trabalho moroso, que pode redundar na destruição da planta (ZEIST et al., 2014). Geralmente os métodos destrutivos exigem a extração da 
folha para a medição da AF, o que impossibilita medições sucessivas na mesma folha (FASCELLA; DARWICH; ROUPHAEL, 2013; KUMAR et al., 2017; SALAZAR et al., 2018).

Os métodos indiretos têm como vantagens a maior rapidez e a possibilidade de maior frequência de mensuração, além de apresentar resultados satisfatórios desde que calibrados corretamente (COELHO FILHO et al., 2012). Além disso, muitos métodos indiretos não são destrutivos, sendo, por isso, amplamente utilizados na literatura (KERAMATLOU et al., 2015; SALA et al., 2015; KUMAR et al., 2017; LIU et al., 2017; SALAZAR et al., 2018). Os métodos não destrutivos, em sua maioria, fazem uso de um conjunto complexo de medidas e modelos matemáticos, ou então da análise digital de fotografias (SPANN; HEEREMA, 2010). Apesar da rapidez e da facilidade de execução, métodos não destrutivos incorrem em grandes erros (COELHO FILHO et al., 2005) quando não calibrados, além de terem sua validade restringida a um determinado genótipo (SPANN; HEEREMA, 2010).

Um método indireto simples de estimativa da AF é o método das dimensões. Trata-se de um método não destrutivo, considerado preciso, em que a AF é determinada por meio da relação entre o comprimento e a largura do limbo foliar, ponderada por um fator de forma (f) (KUMAR et al., 2017; LUCENA et al., 2018). Esse é um método que propicia a mensuração no campo, sem causar danos às plantas, de maneira rápida, sendo o fator de forma de fácil obtenção.

O método da imagem digital por fotografia (ECHEVERRIA et al., 2015) e o método da imagem digital por scanner (ZEIST et al., 2014; SALA et al., 2015) são métodos diretos de obtenção da AF, e, apesar de considerados satisfatórios (ZEIST et al., 2014; KOUBOURIS et al., 2018), são métodos destrutivos e/ou necessitam de instrumentos e softwares, que geralmente são de alto custo (KUMAR et al., 2017). Felizmente, alternativas gratuitas como o software ImageJ tornam o método da imagem digital por scanner viável.

As técnicas planimétricas (KERAMATLOU et al., 2015), apesar de simples, são destrutivas e morosas, principalmente em folhas com bordas irregulares. Apesar disso, essa técnica ainda é considerada como padrão (LEITE et al., 2017) na obtenção da AF, e em virtude disso os resultados obtidos por métodos alternativos foram confrontados com os obtidos pelo método planimétrico (IGATHINATHANE et al., 2006).

A escolha do método depende do objetivo, do grau de precisão desejado, do tamanho da amostra, da morfologia das folhas, dos equipamentos disponíveis, dos custos envolvidos e do tempo de execução das atividades de medição (COELHO FILHO et al., 2005). Poucos estudos compararam a equivalência desses métodos, especialmente no que tange aos métodos destrutivos e aos não destrutivos. Essa comparação torna-se de extrema importância na escolha do método e para embasar a substituição de métodos destrutivos em estudos que visem ao acompanhamento do crescimento e da expansão foliar (ZEIST et al., 2014; KERAMATLOU et al., 2015). Outra importante questão é que estudos relativos à área foliar em espécies nativas como o urucum são escassos.

Nesse contexto, o presente estudo teve como objetivo comparar diferentes métodos para a estimativa da área foliar em mudas de urucum (Bixa orellana L.). Os métodos contemplados foram o método digital por scanner, o método das dimensões foliares e o método planimétrico.

\section{Material e métodos}

\section{Local e protocolo experimental}

O experimento foi conduzido no Viveiro Florestal "Luiz Fernando Oliveira Capellão", pertencente ao Instituto de Florestas, localizado na Universidade Federal Rural do Rio de Janeiro (UFRRJ), município de Seropédica (latitude: $22^{\circ} 46^{\prime} 27^{\prime \prime S}$, longitude: $43^{\circ} 41^{\prime} 10^{\prime \prime} \mathrm{O}$ e altitude $26 \mathrm{~m}$ ), no estado do Rio de Janeiro. O clima de Seropédica, segundo a classificação climática de Köppen-Geiger, é tropical megatérmico com estação seca no inverno (Aw) (ALVARES et al., 2013).

Para a produção das mudas, as sementes foram inicialmente embebidas em água destilada para a quebra da dormência, processo que leva em torno de 10 horas, conforme recomendado em 
Ferreira Filho (2018). As sementes que flutuaram foram descartadas. Depois dessa etapa, realizouse a semeadura manual em 10 de dezembro de 2017, colocando-se cada semente em uma cova de aproximadamente $2 \mathrm{~cm}$ em tubetes de $50 \mathrm{~cm}^{3}$. Os tubetes foram dispostos em seis bandejas com dimensões de $58,2 \mathrm{~cm}$ x 41,0 cm x 16,5 cm, com 187 células para tubetes. As mudas foram espaçadas de forma que totalizassem 51 mudas em cada bandeja.

$\mathrm{O}$ substrato utilizado foi um produto comercial à base de casca de pinus compostada e vermiculita. Esse substrato recebeu fertilização de base segundo recomendação de Gonçalves et al. (2000) com as seguintes doses por $\mathrm{m}^{3}$ de substrato: $150 \mathrm{~g}$ de $\mathrm{N}$ por meio do uso de sulfato de amônio, 300 $\mathrm{g}$ de $\mathrm{P}_{2} \mathrm{O}_{5}$ por meio do uso de superfosfato simples, $100 \mathrm{~g}$ de $\mathrm{K}_{2} \mathrm{O}$ por meio do uso de cloreto de potássio, e $150 \mathrm{~g}$ de FTE Br12 (1,8\% de B, 0,8\% de $\mathrm{Cu}, 3,0 \%$ de Fe, $2,0 \%$ de $\mathrm{Mn}$ e $0,1 \%$ de $\mathrm{Mo}$ ) para fornecimento de micronutrientes. A fertilização de cobertura foi realizada de acordo com a recomendação de Gonçalves et al. (2000), sendo composta de $200 \mathrm{~g}$ de $\mathrm{N}$ por meio do uso de sulfato de amônio, e $180 \mathrm{~g}$ de $\mathrm{K}_{2} \mathrm{O}$ por meio do uso do cloreto de potássio, para 100 litros de solução nutritiva, aplicando-se $5 \mathrm{ml}$ por muda. A primeira fertilização de cobertura ocorreu após 30 dias da semeadura, repetindo-se a cada quinze dias para a fertilização nitrogenada e a cada 30 dias para a fertilização potássica.

Os recipientes durante a produção das mudas permaneceram em canteiros suspensos em pleno sol, sendo realizadas irrigações diárias de duas a três vezes, num volume diário de $15 \mathrm{~mm}$ pelo sistema de aspersão.

Das seis bandejas experimentais preparadas, quatro bandejas foram destinadas à avaliação dos métodos: planimétrico, em que oito mudas foram utilizadas; das dimensões foliares, em que 40 mudas foram utilizadas; e do scanner, em que 40 mudas foram utilizadas. As duas bandejas remanescentes foram usadas para a reposição das mudas retiradas semanalmente para as avaliações dos métodos destrutivos. As determinações de área foliar $(\mathrm{AF})$ foram realizadas semanalmente no período de 22 de março até 10 de maio de 2018 (período de outono), o que totalizou oito semanas, 37 mudas e 229 folhas (Tabela 1). Para os meses de março, abril e maio (até 10 de maio) de 2018, houve precipitação acumulada de $113 \mathrm{~mm}, 83 \mathrm{~mm}$ e $16 \mathrm{~mm}$ e as médias de temperatura foram de $26,8^{\circ} \mathrm{C}, 24,2^{\circ} \mathrm{C}$ e $23,3^{\circ} \mathrm{C}$, respectivamente. As informações meteorológicas foram obtidas na estação do Instituto Nacional de Meteorologia, localizada em Seropédica (Seropédica - Ecologia Agrícola (RJ), Código OMM: 86878), distante cerca de $1150 \mathrm{~m}$ do local do experimento.

\section{Tabela 1 - Datas das campanhas de medidas e número de plantas e de folhas para cada método avaliado}

Table 1 - Measurement dates, number of seedlings and leaves used for each method

\begin{tabular}{ccccc}
\hline Método & \multicolumn{2}{c}{ Planimétrico } & \multicolumn{2}{c}{ Dimensões/Scanner } \\
\hline Data & Mudas & Folhas & Mudas & Folhas \\
\hline $\mathbf{2 3} / \mathbf{0 3} / \mathbf{2 0 1 8}$ & 1 & 7 & 2 & 16 \\
$\mathbf{2 8} / \mathbf{0 3} / \mathbf{2 0 1 8}$ & 1 & 7 & 5 & 35 \\
$\mathbf{0 5} / \mathbf{0 4} / \mathbf{2 0 1 8}$ & 1 & 6 & 5 & 30 \\
$\mathbf{1 2 / 0 4 / 2 0 1 8}$ & 1 & 6 & 5 & 27 \\
$\mathbf{1 9 / 0 4 / 2 0 1 8}$ & 1 & 5 & 5 & 28 \\
$\mathbf{2 6 / 0 4 / 2 0 1 8}$ & 1 & 6 & 5 & 29 \\
$\mathbf{0 3} / \mathbf{0 5} / \mathbf{2 0 1 8}$ & 1 & 7 & 5 & 31 \\
$\mathbf{1 0 / 0 5 / 2 0 1 8}$ & 1 & 6 & 5 & 33 \\
\hline Total & 8 & 50 & 37 & 229 \\
\hline
\end{tabular}

Fonte: Autores (2019) 
Para a determinação da AF pelos métodos, selecionaram-se aleatoriamente cinco mudas para cada campanha de medida, com exceção da primeira campanha, com duas mudas. A área foliar da muda 1 foi determinada por todos os métodos (planimétrico, dimensões e scanner), enquanto a $\mathrm{AF}$ de todas as folhas das mudas 2, 3, 4 e 5 foram determinadas pelo método das dimensões e o método do scanner. As mudas retiradas para análise destrutiva foram repostas (duas bandejas para reposição) e, assim, manteve-se o número de mudas para a determinação da AF constante nas bandejas durante o experimento.

Por fim, foram consideradas apenas as folhas completamente expandidas com pelo menos um centímetro de comprimento (MARTINS et al., 2008; ABREU et al., 2015).

\section{Método da imagem digital por scanner}

No método da imagem digital por scanner, as folhas das cinco mudas selecionadas aleatoriamente foram digitalizadas separadamente para fins de organização. Todas as folhas foram separadas semanalmente, identificadas e colocadas sobre um scanner de mesa do modelo $\mathrm{HP}^{\circledR}$ Deskjet $\mathrm{F} 4280$, acoplado a um computador, junto a uma régua para digitalização (régua de calibração em centímetros). As imagens escaneadas em resolução de $200 \mathrm{dpi}$, em preto e branco foram salvas na extensão "jpg" e processadas pelo software ImageJ (ZEIST et al., 2014; KOUBOURIS et al., 2018) para a obtenção da AF.

\section{Método das dimensões foliares}

O método das dimensões foliares é um método destrutivo e parte da sua execução foi feita no Laboratório de Pesquisa em Reflorestamento (LAPER) da UFRRJ. No LAPER, todas as folhas selecionadas foram destacadas, identificadas e separadas para as medidas das variáveis comprimento $(\mathrm{C})$ e largura (L), com o uso de um paquímetro digital. O comprimento foi medido ao longo da nervura principal e a largura de forma perpendicular à inserção do pecíolo no limbo (SALA et al., 2015).

Após a identificação e determinação das dimensões das folhas, elas foram escaneadas e sua área determinada pelo software ImageJ. As informações sobre as dimensões (C e L) e área foliar (AF) de cada folha foram pareadas, e o fator de forma "f" foi determinado pela análise de regressão linear simples (modelo com e sem intercepto) e de regressão potencial (LUCENA et al., 2018). A AF obtida via ImageJ foi empregada como variável resposta $\left(\mathrm{Y}, \mathrm{cm}^{2}\right)$ e o produto de suas dimensões $(\mathrm{C} \times \mathrm{L})$ como variável preditora $\left(\mathrm{X}, \mathrm{cm}^{2}\right)$. Foram utilizadas as mudas $2,3,4$ e 5 . Na regressão linear, o coeficiente angular da reta de regressão $\left(\beta_{1}\right)$ corresponde ao "f", usado como ponderador para a obtenção da AF em relação ao produto entre o comprimento e a largura da folha ( $\mathrm{AF}=\mathrm{C} \times \mathrm{L}$ x f). A calibração desse método foi feita com 179 folhas. Os modelos lineares empregados, com e sem intercepto, estão representados pelas equações 1 e 2 , respectivamente, enquanto que o modelo potencial está representado na equação 3:

$$
\begin{aligned}
& A F_{\text {est }}=\beta_{0}+C \cdot L \cdot f \\
& A F_{\text {est }}=C \cdot L \cdot f \\
& A F_{\text {est }}=a+(C \cdot L)^{b}
\end{aligned}
$$

Em que: $\mathrm{AF}_{\text {est }}$ é a área foliar estimada; C.L é o produto entre comprimento e largura da folha; $f$ é o fator de forma $\left(\beta_{1}\right) ; \beta_{0}$ é o intercepto do modelo; "a" e "b" são parâmetros do modelo, a serem estimados. 


\section{Método planimétrico}

O método planimétrico consistiu em desenhar sobre o papel milimetrado o contorno de todas as folhas da muda, que foram selecionadas para tal metodologia. Foi utilizada uma muda por campanha para se obter a área foliar pelo método planimétrico, totalizando oito mudas e 50 folhas. A AF foi determinada por meio da contagem e soma das maiores $\left(\mathrm{cm}^{2}\right)$ e menores unidades $\left(\mathrm{mm}^{2}\right)$ de áreas no papel milimetrado. O método planimétrico foi considerado o método padrão (LEITE et al., 2017).

\section{Análise estatística}

O primeiro passo foi verificar qual modelo de regressão (linear com ou sem intercepto e potencial) melhor estima a AF pelo método das dimensões em relação à AF do scanner. Para tal, considerou-se o coeficiente de determinação $\left(\mathrm{R}^{2}\right)$, o erro percentual absoluto médio (EPAM) e a raiz do quadrado médio do erro (RQME) entre a AF pelo método do scanner (variável dependente) e a AF pelo método das dimensões (variável independente). A regressão que proporcionou o maior $\mathrm{R}^{2}$ e os menores EPAM e RQME foi escolhida para representar o método das dimensões.

Logo após a determinação do melhor modelo para representar o método das dimensões, foi feita a análise e a comparação entre os métodos considerados alternativos (dimensões e scanner) e o método padrão (planimétrico). Nessa etapa, considerou-se apenas a área foliar de todas as folhas da muda 1, na qual foram aplicados todos os métodos. Os procedimentos estatísticos usados para verificar a equivalência entre os métodos de estimativa da AF foram o teste $\mathrm{F}$, teste $\mathrm{t}$ e a regressão linear simples, sem intercepto $\left(\mathrm{Y}=\beta_{1} \bullet \mathrm{X}\right)$. $\mathrm{O}$ teste $\mathrm{F}$ e o teste $\mathrm{t}$ foram aplicados aos dados de AF estimada pelos diferentes métodos, em cada campanha de medição, para verificar a igualdade das variâncias e das médias, respectivamente. Já na análise de regressão linear, consideraram-se todas as campanhas de medição, em que a AF pelo método considerado padrão constituiu o eixo das abscissas $(\mathrm{X})$ enquanto que as estimativas da área foliar pelos métodos alternativos constituíram o eixo das ordenadas $(\mathrm{Y})$. A análise de regressão foi utilizada com a expectativa de obtenção de uma relação 1:1 entre as estimativas de AF dos métodos comparados, o que proporcionaria um coeficiente angular igual a $45^{\circ}\left(\beta_{1}=1\right)$. O teste $\mathrm{t}$ foi utilizado para testar a hipótese nula $\left(\mathrm{H}_{0}\right): \beta_{1}=1$, em detrimento da hipótese alternativa $\left(\mathrm{H}_{1}\right): \beta_{1} \neq 1$.

Para a avaliação e comparação do desempenho dos diferentes métodos para a estimativa da AF, foram calculadas estatísticas de precisão e exatidão, como: o índice BIAS, o coeficiente de correlação $(\mathrm{R})$, o coeficiente de determinação $\left(\mathrm{R}^{2}\right)$, o índice de concordância de Willmott (d) e o índice de desempenho (c), conforme descrito em Martins et al. (2014) e os índices de erro (EPAM e RQME).

O EPAM (\%) e a RQME $\left(\mathrm{cm}^{2}\right)$ podem ser obtidos de acordo com Liu et al. (2017) e Sala et al. (2015), respectivamente.

\section{Resultados e discussão}

\section{Ajuste do fator ' $f$ ' das dimensões foliares}

Os modelos de regressão linear e o modelo potencial empregados na obtenção do fator "f" considerado no método das dimensões foliares estão representados na Figura 1. Todos os parâmetros estimados foram significativos estatisticamente pelo teste t a $5 \%$ de probabilidade de erro, com exceção do parâmetro $\beta_{0}$ (intercepto) do modelo linear. De maneira geral, não houve diferença estatística na predição pelos diferentes modelos devido à proximidade entre os índices $\mathrm{R}^{2}$, EPAM e RQME, o que diverge de resultados obtidos em outros estudos que encontraram 
vantagens no modelo potencial (SILVA et al., 2017; LUCENA et al., 2018). Os ajustes foram considerados excelentes, o que atestou a capacidade de predição da AF, devido aos valores elevados de coeficiente de determinação $\left(\mathrm{R}^{2}>0,98\right)$ e aos baixos índices de erro (EPAM $<7 \%$ e RQME $<0,77 \mathrm{~cm}^{2}$ ). Por se tratar de um modelo mais simples e com excelente desempenho (parcimônia), definiu-se o fator de forma para a estimativa da área foliar de mudas de urucum como 0,64 , relativo ao modelo linear sem intercepto.

Figura 1 - Modelos de regressão linear com intercepto (painel a), sem intercepto (painel b) e potencial (painel c) utilizados para estimativa da área foliar de urucum, pelo método das dimensões

Figure 1 - Regression linear models with intercept (panel a), without intercept (panel b) and potential (panel c) used to estimate the leaf area of Urucum, using the method of dimensions

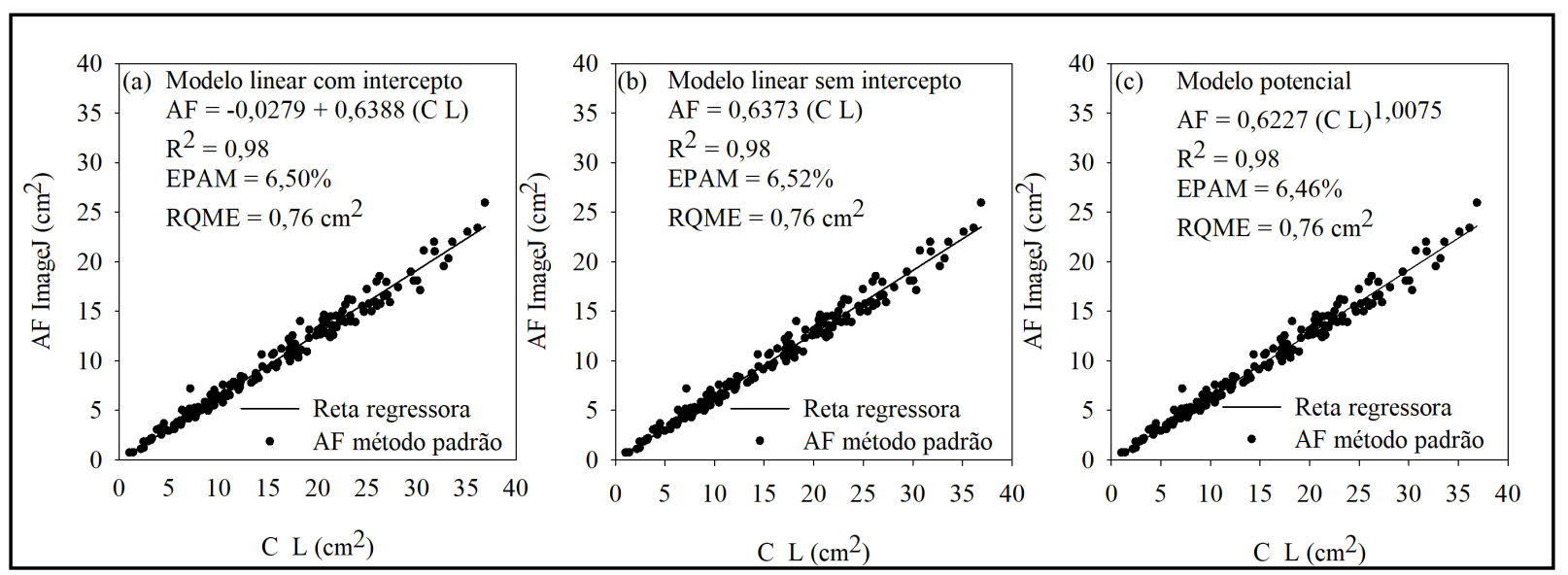

Fonte: Autores (2019)

O fator de forma estimado $(0,64)$ neste estudo foi próximo ao valor determinado por Moraes, Bianco e Araujo (1993), de 0,66, para estimar a área foliar do urucuzeiro (Bixa orellana L.), por meio do produto do comprimento $(\mathrm{C})$, da largura (L) e fator de forma. Contudo, o $\mathrm{R}^{2}$ obtido pelos autores $(0,88)$ foi inferior ao observado no presente trabalho $(0,98)$. Na estimativa do fator de forma de cinco cultivares de maçãs, Sala et al. (2015) obtiveram $\mathrm{R}^{2}$ superiores a 0,99, compatíveis com os encontrados neste estudo, o que confirmou a linearidade do método. Quando comparado com o fator de forma de outras espécies, o urucuzeiro apresentou menor valor em relação aos obtidos para a macieira (f entre 0,69 e 0,79) (SALA et al., 2015), para a mangueira (f $=0,71$ ) (LIMA et al., 2012), para espécies florestais como o Eucalyptus grandis, Eucalyptus saligna e o Corymbia citriodora ( $\mathrm{f}=0,73,0,77$ e 0,72 , respectivamente) (MARTINS et al., 2008; ABREU et al., 2015) e cultivares de oliveira ( $\mathrm{f}=0,71$ - para o modelo linear utilizando o comprimento e a largura) (KOUBOURIS et al., 2018). Porém, o valor de f foi próximo ao obtido para a lichia $(0,66)$ (OLIVEIRA et al., 2017). O menor valor do fator de forma para o urucum em relação ao obtido para outras espécies tem relação com a morfologia de suas folhas, com ápice acuminado e base cordada. Isso reforça a necessidade de estudos como este, que visam estabelecer fatores de forma específicos, uma vez que existem diferenças expressivas entre eles, devido à morfologia foliar particular das espécies.

A Figura 2 apresenta os valores máximos, mínimos (área), médios (circunferência) e o desvio padrão (barra vertical) de cada método de obtenção de área foliar, em cada campanha. É possível observar que essas estatísticas de posição (valores máximos, mínimos e médios) e de dispersão (desvio padrão) foram muito próximas entre os três métodos, em todas as campanhas de medida. O teste F não apontou diferença nas variâncias das áreas foliares, em cada campanha, 
entre os métodos de estimativa de área foliar, a 5\% de probabilidade de erro. Da mesma forma, o teste $\mathrm{t}$ não apontou diferenças entre as médias de AF obtidas pelo método padrão (planimétrico) e pelos métodos alternativos (método das dimensões foliares e scanner) em cada campanha de determinação da área foliar. A análise em cada campanha mostrou que houve similaridade nos resultados de área foliar pelos diferentes métodos, o que indicou a possibilidade de escolha do melhor método, de acordo com o objetivo do estudo, sem prejuízos.

\section{Figura 2 - Valores máximos, mínimos, médios e desvios padrões das estimativas de área foliar em cada campanha, obtido por diferentes métodos}

Figure 2 - Maximum, minimum, mean and standard deviations of leaf area estimates in each campaign, obtained by different methods

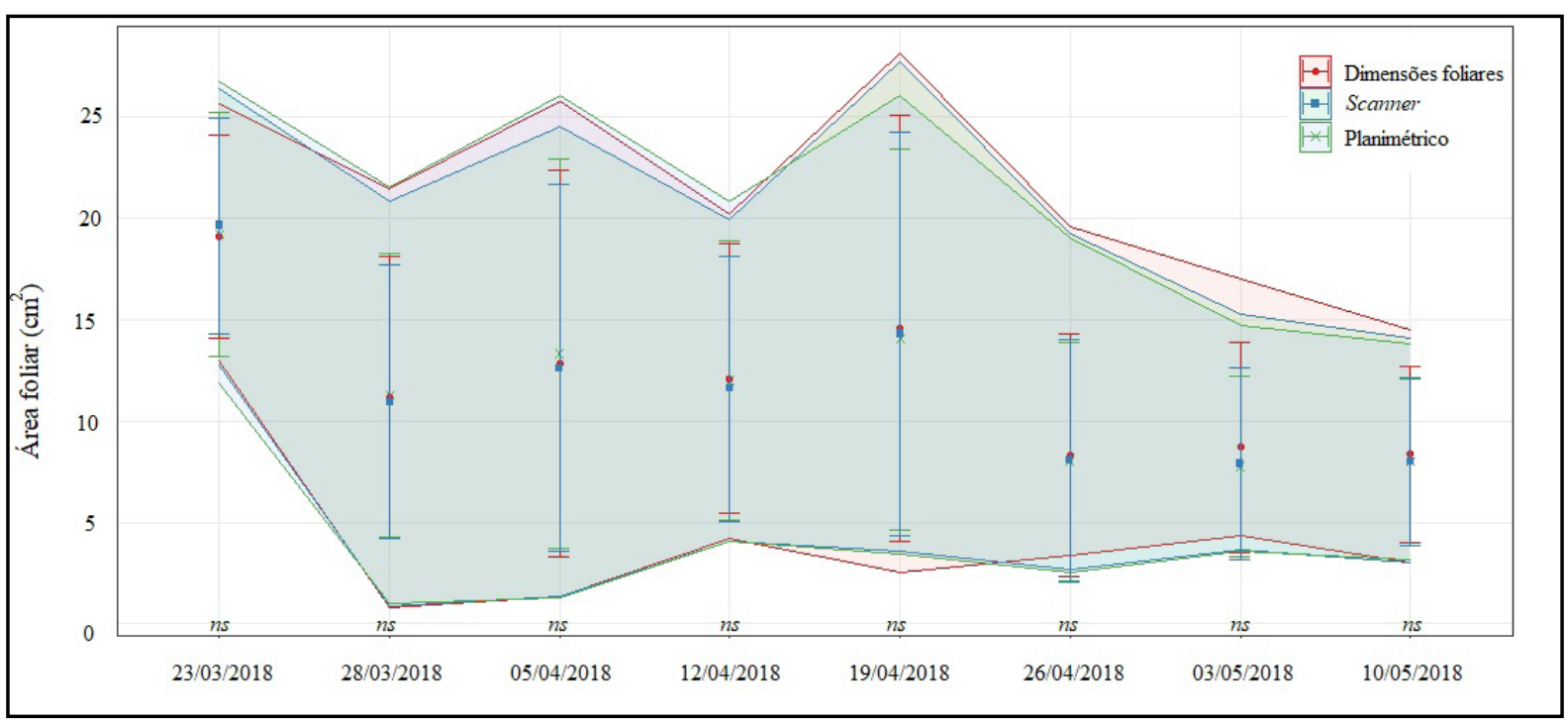

Fonte: Autores (2019)

Em que: $n s$ = diferença estatística não significativa entre as médias e as variâncias obtidas pelos diferentes métodos de obtenção de área foliar.

A Tabela 2 mostra os valores de $\beta_{1}$, do teste $\mathrm{F}$ da regressão, os $\mathrm{p}$-valores do teste $\mathrm{t}\left(\mathrm{H}_{0}: \beta_{1}=\right.$ 1) e os intervalos de confiança para $\beta_{1}$ obtidos na análise de regressão entre o método padrão e os métodos alternativos. Há equivalência entre os métodos alternativos e o método padrão (p-valor $>0,05)$, uma vez que, a um nível de significância de $5 \%$, não houve evidências suficientes para rejeitar a hipótese $\mathrm{H}_{0}$ de que $\beta_{1}=1$. Em termos práticos pode-se admitir a substituição do método padrão (planimétrico), que é extremamente trabalhoso, por métodos de execução mais simples e com concordância na estimativa da AF. Porém, após se compararem os métodos alternativos, a equivalência só foi confirmada a um nível de significância de $1 \%$ (maior probabilidade de erro tipo 2). Apesar disso, os métodos alternativos são extremamente semelhantes em termos de resultados, podendo ser aplicados sem comprometimento da estimativa da $\mathrm{AF}$.

Com base no $\beta_{1}$, pode-se inferir que o método das dimensões superestimou em torno de $0,86 \%$ a AF, enquanto que o método do scanner (ImageJ) a subestimou em torno de 0,76\%. Esses desvios podem ser considerados irrelevantes em estudos de área foliar, o que confirma a equivalência entre os métodos de estimativa da AF. A dispersão dos dados, a reta de regressão e o $\mathrm{R}^{2}$ de cada comparação estão representados na Figura 3. Observou-se pequena dispersão dos dados em relação à reta de concordância (reta 1:1). Além disso, o $\mathrm{R}^{2}$ foi superior a 0,98 na comparação dos diferentes métodos, o que revelou a linearidade entre as estimativas. Essas evidências sustentam a equivalência obtida pela análise de regressão e corroboram o fato de 
que, para o urucum, a utilização de diferentes métodos de obtenção de AF gerou resultados em conformidade com o método padrão.

Tabela 2 - Teste de hipóteses para o coeficiente $\beta_{1}: H_{0}: \beta_{1}=1$ (hipótese nula) e $H_{1}: \beta_{1} \neq 1$ (hipótese alternativa)

Table 2 - Hypothesis test for the coefficient $\beta_{1}: \mathrm{H}_{0}: \beta_{1}=1$ (null hypothesis) e $\mathrm{H}_{1}: \beta_{1} \neq 1$

(alternative hypothesis)

\begin{tabular}{lccccc}
\hline \multirow{2}{*}{ Comparação dos métodos } & $\boldsymbol{\beta}_{\mathbf{1}}$ & \multicolumn{2}{c}{$\mathbf{H}_{\mathbf{0}}: \boldsymbol{\beta}_{\mathbf{1}}=\mathbf{1} ; \mathbf{H}_{\mathbf{1}}: \boldsymbol{\beta}_{\mathbf{1}} \neq \mathbf{1}$} & \multicolumn{2}{c}{ Intervalo de confiança } \\
\cline { 3 - 6 } & & $\mathbf{F}$ & $\mathbf{p}$-valor & Limite inferior & Limite superior \\
\hline Planimétrico x Dimensões & 1,0086 & 1,0982 & 0,2998 & 0,9921 & 1,0251 \\
Planimétrico x Scanner & 0,9924 & 1,6057 & 0,2111 & 0,9805 & 1,0044 \\
Scanner x Dimensões & 1,0160 & 5,9180 & 0,0187 & 1,0028 & 1,0291 \\
\hline
\end{tabular}

Fonte: Autores (2019)

Figura 3 - Análise de coincidência entre o método planimétrico e o método das dimensões (painel a), método planimétrico e método do scanner (painel b) e método do scanner e método das dimensões (painel c) para obtenção de área foliar

Figure 3 - Analysis of coincidence between planimetric method and the method of dimensions (panel a), planimetric method and scanner method (panel b) and scanner method and method of dimensions (panel c) to obtain leaf area

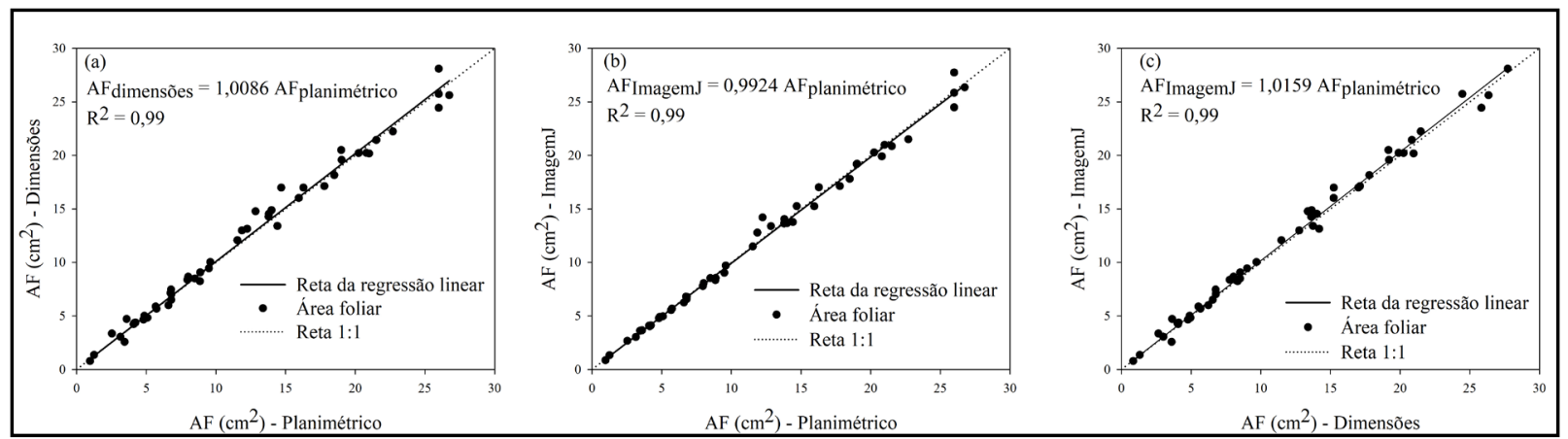

Fonte: Autores (2019)

Flumignan et al. (2008) compararam os métodos das dimensões foliares e do scanner com o método de referência (integrador foliar) na determinação de AF do cafeeiro (Coffea arabica L.). Os autores encontraram valores de coeficiente de determinação e índice d para o método da imagem digital de 0,998 e 0,998, respectivamente. Para o método das dimensões foliares o $\mathrm{R}^{2}$ foi de 0,984 e o d foi de 0,912. Embora essas estatísticas possam ser consideradas ótimas, é preciso ressaltar que o método das dimensões foliares apresentou desempenho inferior em relação ao desempenho observado no presente trabalho. Morgado et al. (2013), ao avaliarem o modelo entre a AF mensurada por dispositivo de medição (medidor de bancada) e a AF estimada pelo modelo de dimensões foliares de espécies de Passiflora sp., encontraram $\mathrm{R}^{2}$ superior a 0,929 e índice "c" superior a 0,942 , o que mostra que as dimensões foliares são um ótimo método para a descrição da área foliar dessas espécies. Outros trabalhos usando o produto das dimensões 
lineares do limbo foliar, por exemplo, para genótipos de cacau (Theobro macacao L.) (SANTOS et al., 2014; SALAZAR et al., 2018), para o morangueiro (Fragaria xananassa Duch.) (ZEIST et al., 2014), para a macieira (Malus domestica Borkh.) (SALA et al., 2015), para a nogueira (Juglans regia L.) (KERAMATLOU et al., 2015) e para a oliveira (Olea europaea L.) (KOUBOURIS et al., 2018), confirmam os resultados obtidos no presente estudo de que o método das dimensões é altamente preciso e eficiente em estimar a AF. Além disso, ressalta-se que o método é de fácil aplicação, com todas as vantagens que métodos não destrutivos apresentam.

O método do scanner também se mostrou equivalente ao método padrão, com excelente desempenho em estimar a AF. Porém, a utilização de métodos destrutivos, como o método do scanner, apresenta como a principal desvantagem a impossibilidade de acompanhamento do crescimento vegetal no tempo (FLUMIGNAN et al., 2008), o que é desejável em estudos agronômicos e fisiológicos. A necessidade de softwares complexos e de custo elevado (LIU et al., 2017) pode ser contornada pelo uso de softwares gratuitos, por exemplo, o ImageJ. Dessa forma o método do scanner torna-se aplicável em diversas culturas, com precisão entre 99,95 e 100\% (SALA et al., 2015), inclusive para o urucum. Porém, é uma alternativa somente para experimentos pequenos, que não necessitem manter a estrutura foliar das plantas.

A Tabela 3 mostra a comparação de cada método alternativo em relação ao método padrão, por meio de estatísticas de ajustes e de índices de erro, além da comparação entre os métodos alternativos. Os valores de variação do índice BIAS entre o método padrão e os métodos alternativos foram próximos a zero, o que deixou claro que os modelos não são tendenciosos. Os coeficientes de correlação foram próximos entre si e próximos a um $(\mathrm{R}>0,99)$, o que evidenciou um alto grau de associação entre a AF estimada pelos métodos testados, sendo a correlação considerada quase perfeita. A exatidão entre os métodos também foi atestada pelo índice de concordância ( $>0,99)$, assim como pelo índice de desempenho geral (c), superior a 0,99, o que permitiu classificar os métodos como tendo "ótimo" desempenho (precisos e exatos). Os índices de erro mostram que os métodos das dimensões e do scanner apresentam erros absolutos (EPAM) inferiores a $7 \%$ e a RQME foi sempre inferior a $0,8 \mathrm{~cm}^{2}$, o que revela que os métodos testados podem ser utilizados sem maiores complicações na determinação da área foliar do urucum. Os métodos alternativos apresentaram diferença estatística mínima entre si (EPAM $<4 \%$ e RQME $\left.<0,59 \mathrm{~cm}^{2}\right)$.

Tabela 3 - Comparação entre métodos de obtenção de área foliar através de estatísticas de ajustes e índices de erro

Table 3 - Comparison between methods of obtaining leaf area through adjustment statistics and error indexes

\begin{tabular}{lcccccc}
\hline \multicolumn{1}{c}{ Estatística de ajuste } & R & BIAS & d & c & EPAM $(\%)$ & RQME $\left(\mathbf{c m}^{2}\right)$ \\
\hline Planimétrico x Dimensões & 0,9943 & 0,0153 & 0,9997 & 0,9939 & 6,5619 & 0,7981 \\
Planimétrico x Scanner & 0,9968 & $-0,0063$ & 0,9998 & 0,9966 & 3,2636 & 0,5850 \\
Scanner x Dimensões & 0,9964 & 0,0217 & 0,9998 & 0,9962 & 5,5814 & 0,6655 \\
\hline
\end{tabular}

Fonte: Autores (2019)

Em que: $\mathrm{R}$ = coeficiente de correlação; BIAS = índice BIAS; $\mathrm{d}$ = coeficiente de Willmott; EPAM = Erro de predição absoluto médio; RQME = Raiz do quadrado médio do erro.

Os erros observados neste estudo podem ser considerados aceitáveis quando comparados com os erros observados em outros estudos que objetivaram avaliar/comparar metodologias de obtenção de área foliar (LIMA et al., 2012; FASCELLA; DARWICH; ROUPHAEL, 2013; 
KERAMATLOU et al., 2015; SALA et al., 2015; KOUBOURIS et al., 2018; SALAZAR et al., 2018). Comparativamente, é possível observar pequena vantagem no método do scanner em relação ao método das dimensões foliares, devido aos menores erros na estimativa de AF, assim como observado por Morgado et al. (2013). Porém, o método das dimensões foliares apresenta vantagens que o tornam atrativo em relação ao método do scanner, tais como ser de baixo custo, de rápida e de fácil aplicação, especialmente em casos em que se exige grande número de medições e/ou medições sucessivas para verificar a dinâmica da AF (ZEIST et al., 2014; KUMAR et al., 2017; SALAZAR et al., 2018).

Portanto, os métodos alternativos testados neste estudo mostraram ter condições de serem usados para a estimativa da AF do urucum. Cabe ao pesquisador, de acordo com os recursos e objetivos de sua pesquisa, escolher o método mais conveniente, uma vez que as opções apresentadas neste trabalho possuem boa precisão e exatidão, com valores próximos aos encontrados pelo método padrão (método planimétrico), podendo, assim, substituí-lo sem prejuízos.

Em função da natureza deste estudo, realizado em um viveiro florestal onde se objetiva produzir mudas mantendo a estrutura foliar intacta, recomenda-se o uso do método das dimensões foliares por não ser destrutivo. A preservação da área foliar na produção de mudas favorece o vigor das mudas, uma vez que a área foliar tem relação direta com a fotossíntese, a produção (FAVARIN et al., 2002; SALA et al., 2015), as trocas gasosas e a transpiração vegetal (MARTINS et al., 2008; ABREU et al., 2015).

\section{Conclusões}

Os métodos das dimensões e do scanner (alternativos) para a estimativa da área foliar do urucuzeiro são precisos e exatos e podem ser usados para a estimativa da área foliar do urucum.

Os métodos das dimensões e do scanner são estatisticamente equivalentes ao método planimétrico (padrão) e a partir deles foram obtidas estimativas da AF próximas entre si. Embora o método do scanner tenha apresentado ajuste superior, o método das dimensões, dependendo da aplicação, pode ser considerado a melhor opção para a estimativa da área foliar, devido ao seu caráter não destrutivo.

\section{Referências}

ABREU, M. C. et al. Valores limítrofes para transpiração, desenvolvimento e crescimento de Corymbia citriodora (Hook.) K.D. Hill \& L.A.S. Johnson em resposta à deficiência hídrica no solo. Revista Árvore, Viçosa, MG, v. 39, n. 5, p. 841-852, set./out. 2015.

ALVARES, C. A. et al. Köppen's climate classification map for Brazil. Meteorologische Zeitschrift, [s. l.], v. 22, n. 6, p. 711-728, dez. 2013.

ARAUJO NETO, A. C. et al. Germination and vigor of Bixa orellana L. seeds pre-soaked in a plant biostimulant. Floresta, Curitiba, v. 48, n. 3, p. 293-302, jun. 2018.

COELHO FILHO, M. A. et al. Estimativa da área foliar de plantas de lima ácida "Tahiti” usando métodos não-destrutivos. Revista Brasileira de Fruticultura, Jaboticabal, v. 27, n. 1, p. 163-167, abr. 2005.

COELHO FILHO, M. A. et al. Método para estimativa do IAF de árvores isoladas ou de plantações com dossel fechado. Revista Brasileira de Engenharia Agrícola e Ambiental, Campina Grande, v. 16 , n. 5 , p. 529-538, maio 2012.

ECHEVERRIA, C. P. et al. Digital Cover Photography for Estimating Leaf Area Index (LAI) in Apple Trees Using a Variable Light Extinction Coefficient. Sensors, Basel, v. 15, n. 2, p. 28602872, jan. 2015. 
FARIA, D. V. et al. Irradiance and light quality affect two annatto (Bixa orellana L.) cultivars with contrasting bixin production. Journal of Photochemistry and Photobiology, Amsterdam, v. 197, p. 1-9, jul. 2019.

FASCELLA, G.; DARWICH, S.; ROUPHAEL, Y. Validation of a leaf area prediction model proposed for rose. Chilean Journal of Agricultural Research, Chillán, v. 73, n. 1, p. 73-76, aug. 2013.

FAVARIN, J. L. et al. Equações para estimativa do índice de área foliar do cafeeiro. Pesquisa agropecuária Brasileira, Brasília, v. 37, n. 6, p. 769-773, jun. 2002.

FLUMIGNAN, D. L. et al. Área foliar de folhas íntegras e danificadas de cafeeiro determinada por dimensões foliares e imagem digital. Coffee Science, Lavras, v. 3, n. 1, p. 1-6, jan./jun. 2008.

FERREIRA FILHO, G. S. Cultivo de urucum: Sistema de produção. Rondônia: EMATER, 2018. $30 \mathrm{p}$.

FONTANA, K. B. et al. Biossorção de $\mathrm{Pb}$ (II) por casca de urucum (Bixa orellana) em soluções aquosas: estudo cinético, equilíbrio e termodinâmico. Química Nova, São Paulo, v. 39, p. 9, p. 1078-1084, jun. 2016.

GONÇALVES, J. L. M. et al. Produção de mudas de espécies nativas: substrato, nutrição, sombreamento e fertilização. In: GONÇALVES, J. L. M.; BENEDETTI, V. (ed.), Nutrição e fertilização florestal. Piracicaba: [s. n.], 2000. p. 309-350.

IGATHINATHANE, C. et al. Interactive computer software development for leaf area measurement. Computers and Electronics in Agriculture, Amsterdam, n. 51, p. 1-16, apr. 2006.

KANDIANNAN, K. et al. Modeling individual leaf area of ginger (Zingiber officinale Roscoe) using leaf length and width. Scientia Horticulturae, Amsterdam, v. 120, n. 4, p. 532-537, 2009.

KERAMATLOU, I. et al. A simple linear model for leaf area estimation in Persian walnut (Juglansregia L.). Scientia Horticulturae, Amsterdam, n. 184, p. 36-39, mar. 2015.

KISSMANN, C.; SCALON, S. P. Q.; TEODOSIO, T. K. C. Condicionamento das sementes e sombreamento na emergência e no crescimento de plantas de Bixa orellana L. Revista de Ciências Agrárias, Recife, v. 36, n. 1, p. 48-56, jan. 2013.

KOUBOURIS, G. et al. Leaf area estimation by considering leaf dimensions in olive tree. Scientia Horticulturae, Amsterdam, v. 240, p. 440-445, oct. 2018.

KUMAR, M. K. et al. Non-destructive estimation of leaf area of durian (Durio zibethinus) - An artificial neural network approach. Scientia Horticulturae, Amsterdam, v. 219, p. 319-325, maio 2017.

LEITE, M. L. M. V. et al. Estimativa da área foliar em of Urochloa mosambicensis por dimensões lineares. Agropecuária Técnica, Areia, v. 38, n. 1, p. 9-16, maio 2017.

LIMA, R. T. et al. Modelos para estimativa da área foliar da mangueira utilizando medidas lineares. Revista Brasileira de Fruticultura, Jaboticabal, v. 34, n. 4, p. 974-980, dez. 2012.

LIU, Z. et al. Non-destructively predicting leaf area, leaf mass and specific leaf area based on a linear mixed-effect model for broadleaf species. Ecological Indicators, Amsterdam, v. 78, p. 340-350, jul. 2017.

LUCENA, L. R. R. et al. Estimativa da área foliar em Urochloa mosambicensis por dimensões foliares e imagens digitais. Archivos de Zootecnia, Córdoba, v. 67, n. 259, p. 408-413, jul. 2018.

MARTINS, F. B. et al. Desenvolvimento foliar em cultivares de oliveira estimados por duas categorias de modelos. Revista Brasileira de Meteorologia, São José dos Campos, v. 29 n. 4, out./dez. 2014. 
MARTINS, F. B. et al. Deficiência hídrica no solo e seu efeito sobre transpiração, crescimento e desenvolvimento de mudas de duas espécies de eucalipto. Revista Brasileira de Ciência Do Solo, Viçosa, MG, v. 32, n. 3, p. 1297-1306, maio/jun. 2008.

MORAES, E. C.; BIANCO, S.; ARAUJO, J. A. Estimativa da área foliar das folhas do urucuzeiro (Bixa orellana L.). Belém: FCAP; EMBRAPA, 1993. 10 p. (Informe técnico, 17).

MORGADO, M. A. D. et al. Estimação da área foliar por método não destrutivo, utilizando medidas lineares das folhas de espécies de Passiflora. Revista Ceres, Viçosa, MG, v. 60, p. 662667, set./out. 2013.

OLIVEIRA, P. S. et al. Leaf area estimation in litchi by means of allometric relationships. Revista Brasileira de Fruticultura, Jaboticabal, v. 39, sup., p. 1-6, fev. 2017.

SALA, F. et al. Leaf area constant model in optimizing foliar area measurement in plants: a case study in apple tree. Scientia Horticulturae, Amsterdam, v. 193, p. 218-224, sep. 2015.

SANTOS, S. N. et al. Análise comparativa de métodos de determinação de área foliar em genótipos de cacau. Bioscience Journal, Uberlândia, v. 30, n. 3, p. 411-419, maio 2014.

SILVA, S. F. et al. Modelos alométricos para estimativa da área foliar de boldo pelo método não destrutivo. Revista Agrarian, Dourados, v. 10, n. 37, p. 193-198, 2017.

SPANN, T. M.; HEEREMA, R. J. A simple method for non-destructive estimation of total shoot leaf area in treefruit crops. Scientia Horticulturae, Amsterdam, v. 125, p. 528-533, maio 2010.

SALAZAR, J. C. S. et al. Non-destructive estimation of the leaf weight and leaf area in cacao (Theobroma cacao L.). Scientia Horticulturae, Amsterdam, v. 229, p. 19-24, feb. 2018.

ZEIST, A. R. et al. Comparação de métodos de estimativa de área foliar em morangueiro. Pesquisa Agropecuária Gaúcha, Porto Alegre, v. 20, n. 1/2, p. 33-41, 2014. 